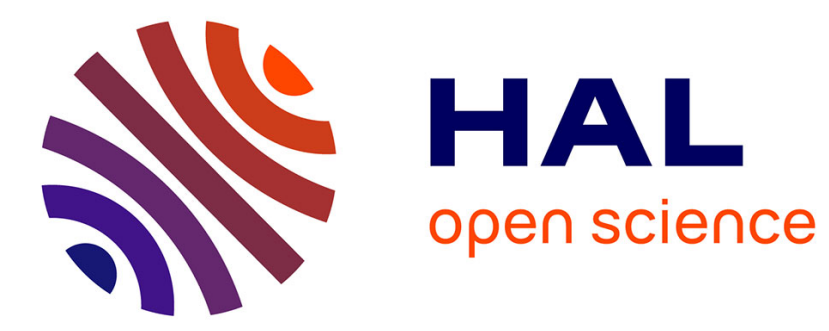

\title{
Polinsar and Tomographic Results Over the Gabonese Forest
}

\author{
Valentine Wasik, Pascale Dubois-Fernandez, Cédric Taillandier, Sassan \\ Saatchi
}

\section{- To cite this version:}

Valentine Wasik, Pascale Dubois-Fernandez, Cédric Taillandier, Sassan Saatchi. Polinsar and Tomographic Results Over the Gabonese Forest. IGARSS 2018 - 2018 IEEE International Geoscience and Remote Sensing Symposium, Jul 2018, VALENCIA, Spain. 10.1109/IGARSS.2018.8518048 . hal-02908469

\section{HAL Id: hal-02908469 \\ https://hal.science/hal-02908469}

Submitted on 29 Jul 2020

HAL is a multi-disciplinary open access archive for the deposit and dissemination of scientific research documents, whether they are published or not. The documents may come from teaching and research institutions in France or abroad, or from public or private research centers.
L'archive ouverte pluridisciplinaire HAL, est destinée au dépôt et à la diffusion de documents scientifiques de niveau recherche, publiés ou non, émanant des établissements d'enseignement et de recherche français ou étrangers, des laboratoires publics ou privés. 


\title{
POLINSAR AND TOMOGRAPHIC RESULTS OVER THE GABONESE FOREST
}

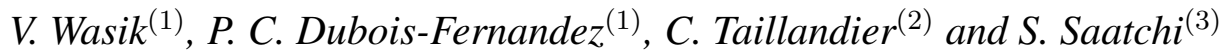 \\ (1): Office National d'Études et de Recherches Aérospatiales, 13661 Salon-de-Provence, France. \\ (2): Total, Centre Scientifique et Technique Jean-Féger, 64000 Pau, France. \\ (3): Jet Propulsion Laboratory, Earth Science Section, California Institute of Technology, Pasadena, CA 91109 USA.
}

\begin{abstract}
The ESA-sponsored AfriSAR campaign took place in Gabon between 2015 and 2016. It was designed to collect data from tropical forests in order to support the future ESA-BIOMASS mission. This paper addresses the potential of P-band PolInSAR and tomography for retrieving vegetation parameters from the multi-baseline airborne data acquired by ONERA over the forest of Lopé. It is shown that a correction of phase disturbances (phase screens) is necessary. A correction procedure based on recent works from the litterature is applied. The PolInSAR and tomographic results are presented and compared with the available LIDAR data.
\end{abstract}

Index Terms - Multi-baseline Synthetic Aperture Radar, SAR Tomography, PolInSAR, Phase Calibration

\section{INTRODUCTION}

The European Spatial Agency (ESA) BIOMASS mission has been selected to be the next Earth Explorer mission: in near future, global observation and monitoring of forests biomass will be ensured by the BIOMASS satellite with a Synthetic Aperture Radar (SAR) system [1]. BIOMASS will be implemented as a P-band SAR system to permit a better penetration in the canopy cover. The system will function in polarization mode with a repeat-pass interferometric orbit and a revisit time of 3-4 days, so that polarimetric and interferometric methods can be implement to analyze the forests parameters.

The ESA-sponsored AfriSAR airborne campaign has been specifically designed to collect data in the context of the BIOMASS mission [2]. The acquisition of the P-band images during this campaign has been conducted in two parts over the tropical forests of Gabon (Africa), firstly in July 2015 with ONERA airborne system SETHI, and secondly in February 2016 with DLR airborne system F-SAR.

SAR interferometry is an interesting image processing technique for the retrieval of vegetation and soil parameters, which can be applied for PolInSAR or tomography. However, phase disturbances originating from uncertainties in trajectories of the airborne platforms create artefacts in the interferometric products. These phase disturbances (also referred as the phase screens) need to be estimated and eliminated from the data to ensure a good interpretation of the PolInSAR or tomographic results.

In this paper, we will focus on the analysis of the Pband multi-baseline data acquired over Lopé National Park by ONERA during the July 2015 AfriSAR campaign. After the implementation of the phase screen correction, PolInSAR heights (ground as well as top of vegetation) and tomographic profiles of forests structure are presented and compared to the available LIDAR data.

\section{ACQUISITIONS OVER LOPÉ}

The airborne SAR system used for the AfriSAR ONERA campaign is the SETHI system, developed by ONERA.

The multi-baseline SAR data set is composed by images acquired at 10 different altitudes over the study site of Lopé. Two LIDAR data sets have also been acquired over the area of Lopé with different instruments. The first set (Small Footprint LIDAR (SFL) data set) was collected by JPL using a Riegl VQ480U sensor in July 2015 with a variable point density and footprint diameter of about $10 \mathrm{~cm}$. A Digital Terrain Model (DTM) and a Canopy Height Model (CHM) were computed from the SFL data. The second set (LVIS data set) was acquired with NASAs airborne Land Vegetation Ice Sensor (LVIS) during March 2016.

\section{MULTI-BASELINE SAR ACQUISITIONS}

\subsection{General background}

We note $s=\left[s_{1}, \ldots, s_{P}\right]^{T}$ the multi-baseline signal, where $s_{p}(p=1, . ., P)$ is the complex backscattered signal received by the plateform $p$ for a polarization channel $X Y$ with $X$ standing for the emitted polarization and $Y$ for the direction of reception $(X, Y=H, V)$. For a point target at altitude $z_{g}$, when no perturbation is present on the propagating path, and after flat-earth pre-processing, the phase difference between the signals $s_{p}$ and $s_{q}$ can be linked to the altitude $z_{g}$ [3]:

$$
\varphi_{p}-\varphi_{q}=k_{z}^{p, q} z_{g}
$$


where $k_{z}^{p, q}$ is the vertical wavenumber for the couple of antennas $p$ and $q$. For a volumetric target such as vegetated areas, the scatterers are distributed between the ground at altitude $z_{g}$ and the top of canopy at altitude $z_{g}+h_{v}$. Considering that the volume is constituted by statistically independent continuous layers, the expected value of the backscattered power received for antenna $p$ can be characterized by [4]:

$$
\left\langle\left|s_{p}\right|^{2}\right\rangle=\int_{z_{g}}^{z_{g}+h_{v}} S(z) d z
$$

where $S(z)=\left\langle|P(z)|^{2}\right\rangle$ refers to the backscattered power of the scatterers at altitude $z$ and $P(z)$ is the complex reflectivity of the medium [5] taking into account the attenuation through the vegetation. Tomographic techniques consist in retrieving the backscattered power $S(z)$ from the multi-baseline acquisitions. In the following, we will use the Capon spectral estimator that gives an estimation $\widehat{S}_{C}(z)$ of the vertical profile.

The PolInSAR estimation approach was chosen to be a single-baseline approach, i.e. that makes use of information from a single couple of antennas from the multi-baseline acquisitions, but for all the polarization channels $X Y$. The estimation method we choose to implement follows the steps described in $[6,7]$. The main difference with the standard estimation procedure proposed by Cloude and Papathanassiou [8] comes from the fact that at P-Band, the ground contribution cannot be neglected, whatever the polarization. As a consequence, the volume only coherence is not accessible directly from the measurements. In order to solve this uncertainty, we assume that the attenuation through the vegetation is $0.4 \mathrm{~dB} / \mathrm{m}$.

\subsection{Phase screens}

The SAR images are frequently perturbed by spacevarying residual phases (phase screens) which can originate from uncertainties in the position of the antennas or from atmospheric perturbations. For point target, it can be represented by an additional term in the phase $\phi_{p}$ the $s_{p}$ coefficient of the multibaseline signal: $\phi_{p}=\varphi_{p}+\alpha_{p}$, where $\alpha_{p}$ is the space-varying phase screen for the image $p$. For a point target of elevation $z_{g}$, the interferometric phase between images $p$ and $q$ can then be written:

$$
\phi_{p, q}=\phi_{p}-\phi_{q}=k_{z}^{p, q} z_{g}+\alpha_{p}-\alpha_{q}
$$

These phase screens can have important consequences on the tomographic and PolInSAR estimations, by emphasing side lobes and defocusing principal lobes of vertical profiles, and by introducing strong biases into PolInSAR estimated ground height. As a consequence, the results are not directly interpretable without phase screen correction. The correction method we choose to apply on the Lopé data is largely inspired by the work of Tebaldini et al. in [9] and will be described in the following section.
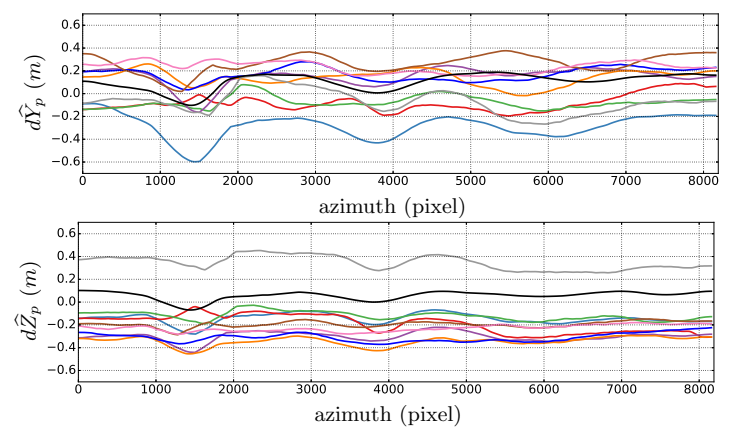

Fig. 1. Estimated trajectory errors $d \widehat{Y}_{p}$ and $d \widehat{Z}_{p}$ as a function of the azimuth position, obtained over Lopé for the 10 flights and after smoothing with a sliding window of 400 pixels large.

\section{PHASE SCREEN ESTIMATION AND DATA CORRECTION}

As recommended in [9], we implement a step of "Phase Linking" [10,9] in order to estimate an ensemble of linked phases using all the available interferograms. For point, surfacic or volumetric targets at altitude $z_{t}$, these linked phases $\left\{\phi_{p, t}^{(\ell)}\right\}_{p=1 . . P}$ for this target can then be expressed as the form of (3) [9]:

$$
\phi_{p, t}^{(\ell)}-\phi_{M, t}^{(\ell)}=k_{z}^{p, M} z_{t}+\alpha_{p}-\alpha_{M}
$$

Under the hypothesis of phase screens originating only from trajectory errors (no atmospheric perturbation for airborne systems), it can be shown that the phase screens $\alpha_{p}$ can be approximated as a function of $d Z_{p}$ and $d Y_{p}$ which represent respectively the position errors of the plateform $p$ in altitude and in the ground range direction for a fixed position in azimuth [9]:

$$
\alpha_{p} \simeq F\left(d Y_{p}, d Z_{p}, \theta\right)=\frac{4 \pi}{\lambda}\left(-d Y_{p} \sin \theta+d Z_{p} \cos \theta\right) .
$$

An estimator of $d Y_{p}, d Z_{p}$ and $z_{t}$ consists in finding the function $F\left(d \widehat{Y}_{p}, d \widehat{Z}_{p}, \theta\right)$ and the value $\widehat{z}_{t}$ that are the most representative of the linked phases difference of (4). For a fixed azimuth position in the image $p$, this step is done by selecting a number $T$ of pixels with different incident angles. The Double Localization iterative procedure described in details in [9] is then put in place. We differ from this method only by the initialisation step by setting $\widehat{z}_{t}^{0}=0$ for $t=1 . . T$. This is a sensible hypothesis for the initialisation since phase calibration of the AfriSAR data has been done using TanDEM-X DEM, which is assumed to be close to the canopy surface.

The estimations of $d Y_{p}$ and $d Z_{p}$ on the Lopé dataset are shown in Figure 1 for the 10 available images, using $T=$ 7 values of $\widehat{\phi}_{p, t}^{(\ell)}$ for the estimation of the $H V$ polarization channel and an initialization mid-azimuth.

The final estimated phase screens are obtained from the 
antennas position errors $d \widehat{Y}_{p}$ and $d \widehat{Z}_{p}$ :

$$
\widehat{\alpha}_{p}=\frac{4 \pi}{\lambda}\left(-d \widehat{Y}_{p} \sin \theta+d \widehat{Z}_{p} \cos \theta\right) .
$$

The multi-baseline data set is then processed by removing the phase screens from each track: $s_{p}^{\text {corr }}=s_{p} e^{-j \widehat{\alpha}_{p}}$, for $p=1 . . P$. The phase screens are supposed not to depend on the chosen polarization channel, only on the errors of plateforms trajectories. Then, the $\widehat{\alpha}_{p}$ obtained from the $H V$ channel are also used for the correction of the other polarization channels. Since the position errors of antennas lead also to erroneous orthogonal baselines between the tracks, the vertical wavenumbers $k_{z}^{p, M}$ are updated accordingly.

\section{RESULTS}

\subsection{Impact of the phase screen correction}

To show the impact of the phase screen correction described in section 4 , the vertical tomographic profiles $\widehat{S}_{C}(z)$ for the SAV2 ROI (savanna region) are shown before and after correction on Figure 2 for the $H V$ channel. The profiles have been normalized regarding to their maxima in the chosen vertical sampling. We superpose the mean SFL DTM on the whole region in black continuous line and the mean canopy SFL elevation in green dashed line, which is simply the sum of the mean DTM and the mean CHM on the whole region. The histograms of ground elevation and canopy elevation are also shown on the tomographic profiles. On Figure 3, the tomographic profiles $\widehat{S}_{C}(z)$ are presented in the azimuth direction for a mid-range section cut after correction for the $H V$ channel (Figure 3(a)) and for the $H H$ channel (Figure 3(b)). The normalization of the profiles has been done for each position in azimuth. The mean SFL DTM and canopy elevation are shown respectively in black continuous and dashed lines. A good correlation with the SFL data can be observed. Moreover, the ground contribution is higher in the $H H$ channel than in the $H V$ channel.

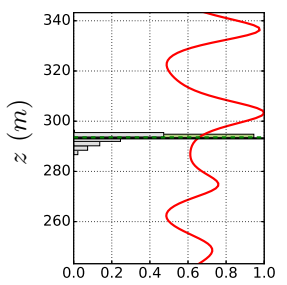

(a) Before correction

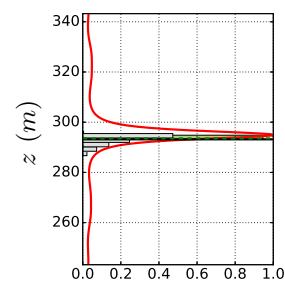

(b) After correction
Fig. 2. In red, normalized tomographic profiles $\widehat{S}_{C}(z)$ on SAV2 for the $H V$ polarimetric channel (a) with and (b) without the phase screen correction described in the section 4 .

\subsection{Comparison with LVIS profiles}

The normalized mean LVIS profiles over 3 other ROIs are presented in blue on Figure 4: OKO2 (Okoumés forest), COL1

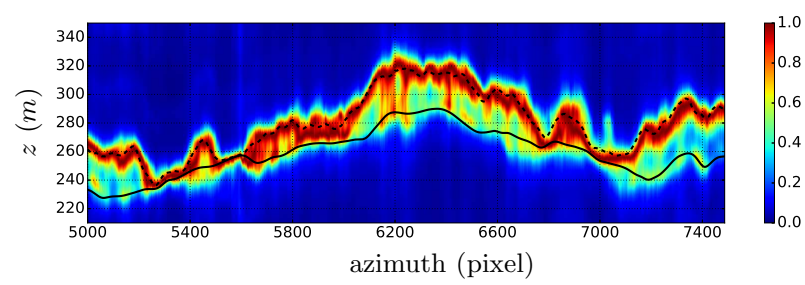

(a) $H V$ after correction

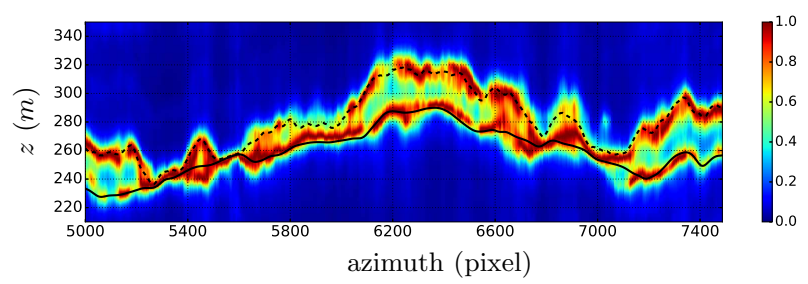

(b) $\mathrm{HH}$ after correction

Fig. 3. Profiles $\widehat{S}_{C}(z)$ in the azimuth direction for a fixed range position in Lopé after phase screen correction. The analysis is done with a $33 \times 33$ pixels sliding window. (a): $H V$. (b): $H H$.

and COL3 (colonizing forests at savannas borders). The corresponding Capon profiles $\widehat{S}_{C}(z)$ for the $H V$ channel are also shown in red. The LVIS and the Capon results show great similarities. Although, SAR tomography in P-band with Capon estimator may fail to produce vertical profiles with a resolution as good as the LVIS imaging system, it always reveals a stronger ground contribution on the studied ROIs.

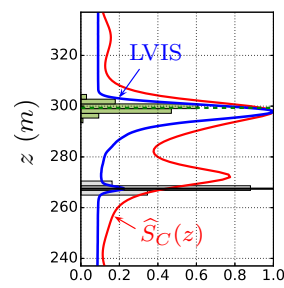

(a) $\mathrm{OKO} 2$

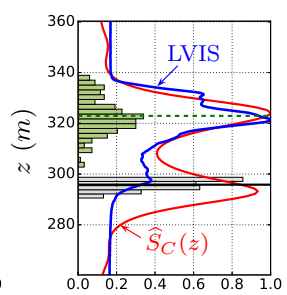

(b) COL1

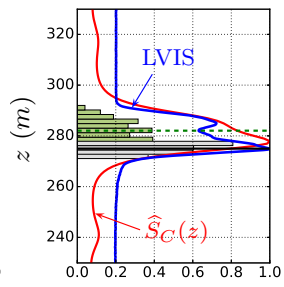

(c) $\mathrm{COL} 3$
Fig. 4. In blue, mean normalized LVIS profiles. In red, normalized Capon profiles $\widehat{S}_{C}(z)$ for the $H V$ channel. ROIs: (a) $\mathrm{OKO} 2$; (b) COL1 ; (c) COL3.

\subsection{PolInSAR results}

The PolInSAR estimation has been performed on the corrected multi-baseline dataset. Figure 5 presents the PolInSAR results for ground height and for vegetation height. The PolInSAR estimated ground height is compared to the TanDEM-X DEM and to the SFL DTM. As expected, the TanDEM-X height is above the SFL ground height: indeed, the X-band phase center height is closer to the top of the vegetation. The PolInSAR ground height agrees well with the SFL DTM as it can be seen on the 2D histogram of Figure 5(f). The vegetation height maps are similar and a quantitative comparison is going to be performed. 


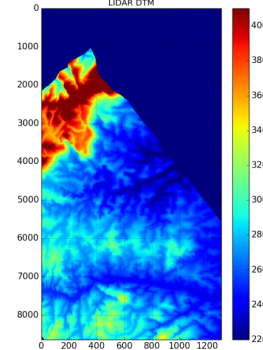

(a) SFL DTM

(d) SFL CHM

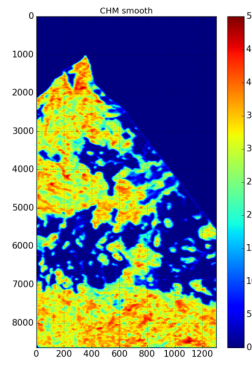

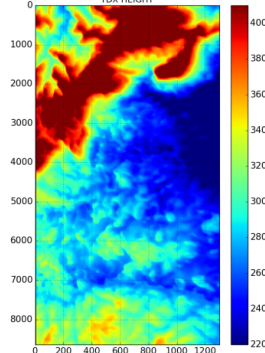

(b) TanDEM-X DEM

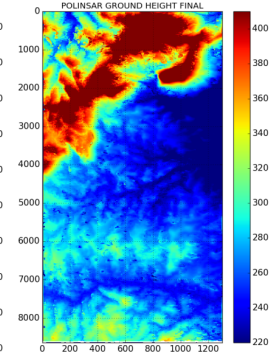

(c) PolInSAR ground height

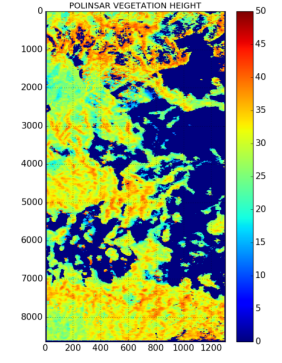

(e) PolInSAR vegetation height

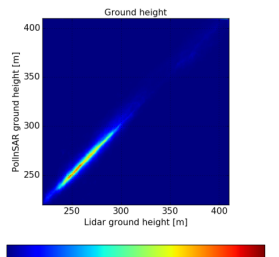

(f)
Fig. 5. (a) SFL DTM, (d) SFL CHM and (b) TanDEM-X DEM in meters over Lopé projected into the geometry of the SAR images acquired by ONERA. PolInSAR estimation results in meters of (c) ground height and (e) vegetation height. (f): 2D histogram of SFL DTM versus PolInSAR estimated ground height.

\section{CONCLUSIONS}

The AfriSAR data collected in Gabon (Africa) in 2015 by ONERA allowed the analysis of the potential of P-band SAR imaging for the ESA-BIOMASS mission in tropical forests. PolInSAR and SAR tomography have been applied on the P-band multi-baseline data acquired on Lopé National Park for the analysis of the forest structure and undercanopy topography. A phase screen correction method based on the Double Localization iterative algorithm proposed by Tebaldini et al. [9] has been applied to remove the residual phases that are perturbing the acquisitions and creating artefacts on the PolInSAR ground height and on the tomographic profiles.

Acknowledgments: The ONERA AfriSAR campaign was supported by ESA, CNES and Total. Forest in situ measurements have been provided by UCL, CESBIO and ANPN. The work at JPL, California Institute of Technology has been carried out under a contract from NASA. The Small Footprint LIDAR data acquisition was supported by the SilvaCarbon project under a grant to University of California, Los Angeles. LVIS data sets were provided by the Laser Vegetation and Ice Sensor team in the Laser Remote Sensing Branch at NASA Goddard Space Flight Center with support from the University of Maryland, College Park.

\section{REFERENCES}

[1] T. Le Toan, S. Quegan, M. W. J. Davidson, H. Balzter, Ph. Paillou, K. P. Papathanassiou, S. Plummer, F. Rocca, S. Saatchi, H. Shugart and L. Ulander, "The BIOMASS mission: Mapping global forest biomass to better understand the terrestrial carbon cycle," Remote Sens. Environ., vol. 115, no. 11, pp. 2850-2860, Nov. 2011.

[2] P. C. Dubois-Fernandez, X. Dupuis, P. Capdessus and R. Baqué, "Preliminary results of the AfriSAR campaign," in Proc. EUSAR 2016: 11th European Conference on Synthetic Aperture Radar, pp. 1-3, Jun. 2016.

[3] R. Bamler and Ph. Hartl, "Synthetic aperture radar interferometry," Inverse Problems, vol. 14, no. 4, pp. R1, 1998.

[4] S. Tebaldini and A. Monti Guarnieri, "On the Role of Phase Stability in SAR Multibaseline Applications," IEEE Trans. Geosci. Remote Sens., vol. 48, no. 7, pp. 2953-2966, Jul. 2010.

[5] S. Tebaldini, "Multibaseline SAR Imaging: Models and Algorithms," Ph.D. dissertation, Dept. Electron. Inf., Politecnico di Milano, Italy, 2009.

[6] P. C. Dubois-Fernandez, J.-C. Souyris, S. Angelliaume and F. Garestier, "The Compact Polarimetry Alternative for Spaceborne SAR at Low Frequency," IEEE Trans. Geosci. Remote Sens., vol. 46, no. 10, pp. 3208-3222, Oct. 2008.

[7] A. Arnaubec, A. Roueff, P. C. Dubois-Fernandez and Ph. Réfrégier, "Vegetation Height Estimation Precision With Compact PolInSAR and Homogeneous Random Volume Over Ground Model," IEEE Trans. Geosci. Remote Sens., vol. 52, no. 3, pp. 1879-1891, March 2014.

[8] S. R. Cloude and K. P. Papathanassiou "Three-stage inversion process for polarimetric SAR interferometry," IEE Proceedings-Radar Sonar and Navigation, vol. 150, no. 3, pp. 125-134, June 2003.

[9] S. Tebaldini, F. Rocca, M. Mariotti d'Alessandro and L. Ferro-Famil, "Phase Calibration of Airborne Tomographic SAR Data via Phase Center Double Localization," IEEE Trans. Geosci. Remote Sens., vol. 54, no. 3, pp. 1775-1792, Mar. 2016.

[10] A. Monti Guarnieri and S. Tebaldini, "On the Explotation of Target Statistics for SAR Interferometry Applications," IEEE Trans. Geosci. Remote Sens., vol. 46, no. 11, pp. 3436-3443, Nov. 2008. 